\title{
MEASUREMENT OF SHORT SPAN STRESS-STRAIN CURVES OF PAPER
}

\author{
Warren J. Batchelor and Bo S. Westerlind
}

\begin{abstract}
A new method is reported for measuring the short span stress-strain curve of paper using a zero/short span tester with additional instrumentation to measure load and displacement during a test. The method is to subtract the zero-span displacement-load curve from the short-span displacement-load curve, so as to obtain the displacement-load curve of the free span. An analysis of the short span test shows that if the sample is well-bonded and the free span is small compared to the fibre length, then the stress-strain behaviour of the free span is independent of span. The new method was used to examine the effect of refining on the short span stress-strain curve of a paper made from an unbleached, never-dried commercial Scandinavian softwood kraft. The breaking strain increased from $12 \%$ for sheets made from the unrefined pulp to $23 \%$ for sheets made from pulp refined to 6,000 PFI revolutions.
\end{abstract}

\section{ADDRESSES OF THE AUTHORS:}

Warren J. Batchelor, Australian Pulp and Paper Institute, Department of Chemical Engineering, Monash University, Australia, and Bo S. Westerlind, SCA Graphic Research AB, Sundsvall, Sweden. Email: warren.batchelor@eng.monash.edu.au

Email: bo.westerlind@research.sca.se

\section{KEYWORDS}

Zero-span test, short-span test, stress-strain curve, residual span

\section{INTRODUCTION}

There have been a number of analytical models for the tensile strength of paper (Jayaraman, Kortschot 1998). All of the models agree that for well-bonded papers, the strength of the fibres is one of the critical factors determining the tensile strength of the sheet. Due to the large number of tests required and the difficulty of the tests, it is very tedious to determine an average fibre strength from testing single fibres. Accordingly, the zero-span tensile strength (ZSTS) has often been used (eg (Page 1969)) as a measure of single fibre strength. This has the added advantage that both the ZSTS and tensile strength are measured on the same set of handsheets and so the state of the fibres under test must be the same in the two measurements. This will not be true when single fibre strengths are used in equations for paper strength, since the state of the fibres will be different in the two tests.

For example, Perez and Kallmes (Perez, Kallmes 1965) measured single fibre strength and compared it with the fibre strength estimated from the ZSTS for twelve pulps using the theory of Van den Akker (Van Den Akker et al. 1958). They found that the single fibre strength estimated from the ZSTS was 30-55\% lower than the measured single fibre strength, which they attributed to fibres with in or out of plane curl not bearing load during the test. In another study, Mohlin et al (Mohlin et al. 1996) showed that the wet zero span strength was a linear function of the number of kinks, twists and angular folds in the fibre. Three such defects produced a 30\% reduction in the ZSTS (Mohlin et al. 1996). The ZSTS also often 
increases after the fibres have been gently refined, as the refining straightens the fibres and removes defects(Seth 1999; Seth 2001).

Any large-scale fibre deformations will reduce the measured zero-span strength, because they reduce the number of fibres that bear load during the test. The alternative in terms of measuring fibre properties of relevance to paper strength would be to measure both the single fibre strength and the state of the fibres within the sheet, including both in and out of plane curl and other defects. This compares with zero-span testing, where all of this information is in some way included because the measured ZSTS is affected by all of these defects. It has been the intent of this brief discussion to demonstrate that in terms of paper mechanical properties the ZSTS is a more relevant measure of fibre strength than that obtained from testing single fibres.

If the whole paper stress-strain curve is to be modeled, and not just the point of fracture, then the stress-strain properties of the fibres must be included. However, as the previous discussion has shown, the fibre stress-strain properties should be measured on the fibres in the sheet, and not by single fibre tests, as this is the only way to guarantee that the state of the fibres is comparable. It has been the goal of the work presented here to develop a method for rapidly measuring, from zero and short span tests, the complete short span stress-strain curve. This will be related to the stress-strain curve of the individual fibres, if bonding does not influence the result at the short spans (up to $0.4 \mathrm{~mm}$ ) that are used. In this paper, we describe the method, and present some results obtained with the method. The relationship between the stress-strain curves measured with the method and the fibre stress-strain curves will be the subject of future communications.

To measure the short-span stress-strain curve, a zero/short span tester was instrumented to allow the load applied to the sample, and the displacement between the jaws, to be measured continuously during each zero or short span test. In order to determine the stress-strain curve, a method has to be found to convert the measured displacements into strains and it is the intent of this paper to present a method for performing this conversion. However, we will first discuss the theory of the zero-span test and show that the required information cannot be obtained from the zero-span test alone.

\section{ZERO SPAN TEST: THEORY.}

At the start of a zero-span test the two sets of jaws are in contact with each other. As the test proceeds an increasing jaw displacement occurs. In the work that we have conducted, the typical displacement at fracture in a zero-span test has been in the range 30-60 $\mu \mathrm{m}$. Despite the status of the zero-span test as an important measurement in the field of paper strength, there has been very little discussion in the literature about the exact mechanisms by which load is transferred to the sample clamped between the jaws and the origin of the measured displacement.

One attempt was made by Cowan (Cowan 1975), who suggested that the displacement arises because the applied load must be transferred by friction from the surface of the jaws to the sample and that the measured displacement is due to slippage under the jaw. Figure 1 shows the forces on the paper under one set of jaws of a zero/short span tester, according to the Cowan friction model. For a given load per unit jaw length, $F_{L}$, Cowan's model assumes (Cowan 1975) that a finite span from the edge of the jaws, determined by the paper-jaw 
coefficient of friction and the applied force, exists before the tensile force in the sample falls to zero. The displacement between the jaws during the test is then due to the straining of this span. In this model, the force in the paper falls from $F_{L}$ at the edge of the jaws to 0 over a distance of $X$. If the clamping pressure is $P_{c}$, then $F_{L}=2 \mu P_{c} X$, where $\mu$ is the paper-jaw coefficient of friction. Thus at any distance, $x(0 \leq x \leq X)$, in from the jaw edge, the tensile force, $F$, on the sample at that position will be given by $F(x)=F_{L}\left(1-2 \mu P_{c} x / F_{L}\right)$.

Analysing the model in more detail we note that if the material is characterised by a constitutive equation, $\varepsilon=f(F)$ then the average strain on the paper under the jaw, $\varepsilon_{a v}$ is given by

$$
\varepsilon_{a v}=\frac{2 \mu P_{c}}{F_{L}} \int_{0}^{F_{L} / 2 u P_{c}} f(F(x)) d x
$$

and the total displacement from the two jaws, $\Delta G_{j}$, is given by $2 \varepsilon_{a v} F_{L} / 2 \mu P_{c}$ and

$$
\Delta G_{j}=2 \int_{0}^{F_{L} / 2 u P_{c}} f(F(x)) d x
$$

If we assume that the short span stress-strain curve can be characterised by some general form $\varepsilon=f(F)=a F+b F^{2}+c F^{3}+\ldots$ then

$$
\Delta G_{j}=\frac{1}{\mu P_{c}}\left[\frac{a F_{L}^{2}}{2}+\frac{b F_{L}^{3}}{3}+\frac{c F_{L}^{4}}{4}+\ldots\right]
$$

Thus if the friction model is assumed to be accurate, $f(F)$ could be theoretically determined by fitting a set of load-displacement data to determine the constants, $a, b, c$ etc. There are a number of practical difficulties with doing so. The first is that, as will be discussed later, zero and short span tests display load take up behaviour at the beginning of the test. This produces a lower slope in the load-displacement curve at the start of the test, making it difficult to accurately fit the data. A further potential difficulty is that the simple analysis presented here is only valid if the compressive stress under the jaws is completely uniform.

\section{NEW METHOD FOR DETERMINING SHORT SPAN PAPER STRESS-STRAIN CURVES}

In a short span test, it must be true that $\Delta G_{t}=\Delta G_{j}+\Delta G_{s}$ where the $\Delta G$ are displacements and the subscripts $t, j$ and $s$ represent the total displacement, the displacement from under the jaws in the zero-span test and the displacement for straining the free span between the jaws, respectively. Thus by subtracting (at the same load) the zero-span from the short-span displacement, the displacement due to straining the free span at that force can be obtained. Repeating this procedure over the whole force range of the test yields a complete set of loaddisplacement data for straining the free span alone, which can be converted to stress-strain, given that the span is known. The utility of this simple method is that it eliminates the need to analyse the mechanical behaviour of the sample under the jaws. The method does assume that the stress distribution through the thickness of the sample is constant at each point in the free span between the jaws.

If we wish to measure a stress-strain curve, which is related to the fibre strength alone, then the measured curve should not depend on fibre length or the bonding between the fibres. This implies that the stress-strain curve should be independent of span. This question is addressed in the analysis given below, which is based on theory in (Batchelor 1999; Batchelor 2002). 
The theory treats the sheet as being composed of load-bearing elements rather than fibres, as fibres may contain gross defects such as kinks and twists across which the fibre is unable to transmit load. Accordingly, it is the number and length of the load bearing elements that influence the mechanical properties of the sheet.

To start with, we consider a load-bearing element, with elastic modulus, $E$, cross-sectional area, $C$, and length, $l$, which spans between the jaws and is at an angle, $\theta$ to the applied stress. . If the overall strain, $\varepsilon$, in the span, $G$, is $\varepsilon=\Delta G / G$, then the component of the force on the fibre in the test direction is

$$
F_{f}=E C \varepsilon \cos ^{3} \theta
$$

If the fibres are randomly oriented then the fibre-orientation probability density function is $2 / \pi$ and the average force on a load-bearing element, $F_{a v}$, can be determined by multiplying Equation 4 by the probability that a fibre will be gripped by both jaws and averaging over the range of possible fibre orientations to yield

$$
F_{a v}=\left(1-\overline{f_{c}}\right) E C \varepsilon \frac{l W_{j}}{A} \frac{2}{\pi} \int_{0}^{\cos ^{-1}(G / l)}\left(\cos ^{4} \theta-\frac{G}{l} \cos ^{3} \theta\right) d \theta
$$

which upon integration and first order Taylor expansion yields

$$
F_{a v}=\left(1-\overline{f_{c}}\right) E C \frac{W_{j}}{A} \frac{3}{8}\left[l-\frac{32}{9 \pi} G\right] \frac{\Delta G_{s}}{G}
$$

where $W_{j}$ is the width of the jaw, $\overline{f_{c}}$ is the fraction of elements, gripped by both jaws that do not bear load due to out-of-plane curl (Perez, Kallmes 1965).

The total number of load-bearing elements in the whole sheet, $\left.I\right|_{o}$, is given by $\left.I\right|_{o}=B A /\left.\omega \bar{l}\right|_{o}$ where $B$ is the sheet basis weight, $A$ is the sheet area, $\omega$ is the average coarseness of the fibres and $\bar{l}_{o}$ is the arithmetic average length of the load-bearing elements. In this theory, a load-bearing element of length, $l$, will bear load only if $l>32 G / 9 \pi$. If the number of loadbearing elements that satisfy this condition is $\left.I\right|_{G}$, and if $G<\left.\bar{l}\right|_{G}$, then $\left.\left.I\right|_{G} \approx I\right|_{o}$ and the average modulus, wall area and length for the set of $\left.I\right|_{G}$ fibres, $\overline{\left.E\right|_{G}}, \overline{\left.C\right|_{G}}$ and $\overline{l_{G}}$ can be replaced by $\overline{\left.E\right|_{o}}, \overline{\left.C\right|_{o}}$ and $\overline{l_{o}}$, respectively. By assuming that for the individual load-bearing elements, $E C$ is largely independent of $l$, we can make the approximation $\overline{\left.\left.\left.E\right|_{o} C\right|_{o} l\right|_{o}} \approx \overline{\left.\left.E\right|_{o} C\right|_{o}} \overline{\left.l\right|_{o}}$. Therefore the force per unit jaw length is

$$
F_{L}=E_{p}\left[1-\frac{32}{9 \pi} \frac{G}{\bar{l} l_{o}}\right] \frac{\Delta G_{s}}{G}
$$

where $E_{p}$ is the modulus at a span length of zero, which by definition is $E_{p}=\left(1-\overline{f_{c}}\right)(3 B / 8 \omega) \overline{\left.\left.E\right|_{o} C\right|_{o}}$. If the fibres in the sheet are bonded to each other, then a fibre that fails to span between the jaws can still transmit some load through the bonds. If the fraction of the load transferred is $c$ then

$$
F_{L}=E_{p}\left(1+(1-c) \frac{32}{9 \pi} \frac{G}{\left.\bar{l}\right|_{o}}\right) \frac{\Delta G_{s}}{G}
$$

Thus if $\left.(1-c)(32 / 9 \pi)(G / \bar{l}]_{o}\right) \approx 0$ then $F_{L}=E_{p} \Delta G_{s} / G$ and the stress-strain curve is independent of the span. Thus in order to accurately determine $E_{p}$ we are faced with two somewhat contradictory requirements, firstly that $(1-c)(32 / 9 \pi)\left(G /\left.\bar{l}\right|_{o}\right)$ be as close to zero as possible and 
secondly that the span, $G$, be as large as possible, maximising $\Delta G_{s}$ and therefore the accuracy of the subtraction. Considering the first requirement, while the average load-bearing element length $\overline{l_{o}}$ is not known, recent work by one of the authors has shown that the average loadbearing element length for five pulps, both laboratory and commercial, softwood and hardwood, was approximately equal to the arithmetic average fibre length (Batchelor 1999; Batchelor 2002). Thus for an unbonded sheet $(c=0)$ of a softwood kraft of arithmetic average fibre length $1.5 \mathrm{~mm}$ and a short span of $300 \mu \mathrm{m}$, the modulus will be $22 \%$ less than at a span of zero. Obviously, for a well-bonded sheet $c \approx 1$ and the difference between the stress-strain curve at a span of zero and at this short span will be negligible.

\section{EXPERIMENTAL}

Force-displacement curves were measured on a Pulmac Zero and short span tensile tester. The tester comes with a computer controlled X-Y table, which allows 24 tests to be performed automatically. The width of the test jaws is $25 \mathrm{~mm}$. The force was calculated from the pressure in the piston driving the jaws apart, using the calibration given by the manufacturer. The separation of the jaws was continuously measured by a Kaman Multi-VIT (Multi-purpose Variable Impedance Transducer). This is a contactless displacement transducer, which was attached to the moving jaw and provided a measurement of position relative to an aluminium target attached to the stationary jaw. The Multi VIT was statically calibrated against a dial gauge. Test data was continuously recorded using PicoLog v5.05.1, and a 12-bit A-D card. The data was collected at a sampling rate of $10 \mathrm{~ms}$, but to reduce noise data was recorded as block averages of five data points.

The material tested in these preliminary studies was handsheets made of an unbleached never-dried softwood kraft pulp from SCA's Östrand mill. Unrefined pulp and pulp refined to 1000,3000 and 6000 revolutions in a PFI mill were used to make handsheets. The Schopper-Riegler freeness was 13.0 for the unrefined pulp and rose to 21.5 after refining for 6000 revolutions in a PFI mill. Handsheets were made under standard conditions except that drying was performed between PTFE sheets, which allow greater shrinkage compared to standard restrained drying. For each sample, 24 tests were conducted at zero span and at spans of 50,101, 159 and $300 \mu \mathrm{m}$. The sheets were tested dry under standard atmospheric conditions.

\section{RESULTS}

\section{Fitting procedures and error sources}

The raw data for 24 tests for one sample are shown in Figure 2. All tests show an initial load take-up behaviour with a lower slope for the load-displacement data at the beginning of the test. It is unclear whether this load take-up is due to slack in the sample or equipment, however the effect was observed for all samples and spans tested. In order for the subtraction technique to be applied successfully, an average curve for all 24 tests in each data set must be calculated. To do this, each of the tests was extracted from the data set and a spline function was fitted to the data set. To remove the effect of load take-up, the maximum slope of each spline fitted curve was determined by linear regression. All data below this point of maximum slope were disregarded. The tangent at the point of maximum slope was then extrapolated to find the displacement at $0 \mathrm{~N}$ force, with the displacement determined in this manner then subtracted from all other displacements. This removes both load take-up effects 
as well as the initial span of the test, if any. Both zero and short span curves corrected in this manner then have a common starting point at $0 \mu \mathrm{m}$ displacement. Each spline fitted curve was then represented as 50 points equally spaced in displacement. The average curve for all 24 tests was then determined by averaging the load and displacement data for each of the 50 points across the 24 tests.

Another important effect, which may influence the accuracy of the fitted curves is to do with the nature of the measurement process. Each of the curves presented here is an average of 24 measurements, which were made on three small disks, each of $6.25 \mathrm{~cm}$ diameter, a test geometry required by the automated sample feeder of the instrument. Thus each curve has been determined from measurements made on three small areas of a sample. If there is systematic strength variation in the samples at this length scale, then this may bias the results. Variation could occur when machine made papers are tested, but it could also occur with handsheets if there is any systematic variation in handsheet grammage across a sheet or between sheets. For such samples it may be necessary to make more measurements to improve the accuracy of the fitted average load-displacement curves.

\section{Unbleached kraft stress-strain curves}

Table 1 summarises all of the measurements, including zero and short span tensile indices and displacements at fracture as well as the strengths and stretch at break from the standard tensile tests. From the table it can be seen that there is no significant difference between the breaking loads measured at the different spans, except for the unbeaten samples. Even for sheets made from the unbeaten fibres, the reduction is small, with the tensile index at fracture falling from $143.9 \mathrm{kNm} / \mathrm{kg}$ at zero span to $132.5 \mathrm{kNm} / \mathrm{kg}$ at a short span of $300 \mu \mathrm{m}$. This difference is just outside the estimated error range of the two measurements. From the theory, this implies that $(1-c)(32 / 9 \pi)\left(G /\left.\bar{l}\right|_{o}\right)<<1$. This is consistent with the samples tested, which were made from a never-dried unbleached softwood kraft. The tensile strength for the unrefined samples was $39 \mathrm{kNm} / \mathrm{g}$, indicating that wet-pressing alone had produced a reasonably well bonded sheet, with high (close to 1.0) values of $c$. While the load-bearing element length, $\left.\bar{l}\right|_{o}$, is not known it should be comparable to the arithmetic average fibre length (Batchelor 1999; Batchelor 2002), since this type of pulp should have relatively few defects. The fibre length was not measured for these samples but based on previous experience, the arithmetic fibre length should be $1.5 \mathrm{~mm}$, five times the largest free span tested here. Thus given that for the samples and spans tested here, $(1-c)(32 / 9 \pi)\left(G /\left.\bar{l}\right|_{o}\right)<<1$, this implies that the stress-strain curve, determined by subtracting the zero span from the short span data sets, will be independent of span, provided that the subtraction can be made with sufficient accuracy.

Figure 3 shows the calculated average load-displacement data for the zero and short span tests on the sheets made from pulp refined to 6000 revs in the PFI mill. The curves should be ordered from left to right, with increasing free span, as the measured displacement should be the sum of the zero and free span displacements. This is not true for the 50 and $100 \mu \mathrm{m}$ span curves, which although they both sit between the 0 and $159 \mu \mathrm{m}$ curves, are reversed from the expected order. The reasons for this discrepancy are not completely clear but must be due to the uncertainties in determining the average curves, which were described previously. 
The stress-strain curves determined by subtraction are shown in Figure 4. The legend indicates the short span curve from which the zero-span curve was subtracted. If the average curves were accurately determined for each span, then after subtraction, all of the stressstrain curves should be coincident. This is clearly not the case for the subtracted curves for the 50 and $101 \mu \mathrm{m}$ spans. It can be concluded that when the span is small, the average loaddisplacement curves cannot be determined with sufficient accuracy for an accurate subtracted curve to be calculated. However, the two curves for the largest spans (159 and $300 \mu \mathrm{m})$ are coincident, as expected. An examination of the displacements at break shown in Table 1 suggests that the subtraction can be accurately made when the difference between the short and zero span curves, at fracture, is $30 \mu \mathrm{m}$ and more.

The subtracted curves for the sheets made from unrefined pulp and pulp refined to $3000 \mathrm{PFI}$ revolutions are shown in Figures 5 and 6, respectively. The subtracted curves for the sheets made from the pulp refined to 1000 PFI revolutions are not shown as they were quite similar to those shown in Figure 6. In neither of Figures 5 and 6 do the stress-strain curves obtained from the different spans completely coincide. This is not surprising when the average displacements at break are examined in Table 1, since only the two $300 \mu \mathrm{m}$ span curves have a final displacement at break, which is $30 \mu \mathrm{m}$ larger than the zero-span displacement. The $159 \mu \mathrm{m}$ short span curves have final displacements at break which are only 18 and $23 \mu \mathrm{m}$ larger than those of the corresponding zero span curves, for the sheets made from unbeaten pulps and pulps beaten to 3000 PFI revolutions, respectively. The difficulties of performing the subtraction when the differences in displacement are small can be particularly seen for the 50 and $100 \mu \mathrm{m}$ curves on these two graphs. These curves show frequent small changes in slope, both increasing and decreasing. Some curves (e.g. the $101 \mu \mathrm{m}$ curve in Figure 6) have sections where the calculated strain stays constant with increasing load. These difficulties could possibly be overcome by improving the accuracy of the calculated average curves by increasing the number of samples tested and improving the fitting process.

The effect of PFI mill refining on the stress-strain curve is plotted in Figure 7. The data plotted here is the subtracted $300 \mu \mathrm{m}$ span curves, since these should be the most accurate. All four curves show a small change of slope around $40 \mathrm{kNm} / \mathrm{kg}$. However, these slope changes are probably only artefacts of the process used to calculate the stress-strain curves, as $40 \mathrm{kNm} / \mathrm{kg}$ is approximately the point of maximum slope in all of the original data sets. As mentioned previously all measurements display load take-up behaviour, which was removed during fitting by calculating the point of maximum slope and discarding all data under this point.

The curves in Figure 7 show clear differences with the level of refining. The short span stress-strain curve for the sheets made from the unrefined pulp is apparently linear-elastic until shortly before fracture. The yield stress is approximately $120 \mathrm{kNm} / \mathrm{kg}$. The tensile index at fracture increases after refining for 1000 PFI revs and then remains constant with increased refining. This is probably due to the refining process straightening fibres, and removing defects, thus increasing the number of fibres bearing load at fracture and increasing the strength (Seth 2001). The stress-strain curve for the sheets made of pulp refined to 6000 PFI revs shows a much lower modulus than the curves for sheets made from the other three pulps. The fracture strain was calculated to be $23 \%$ compared to $12 \%$ for the unrefined sample. The stress-strain curve for the sheets made with pulp refined to 1000 and 3000 PFI revolutions both have a similar initial modulus to the unrefined pulp and remain linear until shortly before fracture. The increase in the strain at fracture for the sheet made of fibres 
refined to 6000 PFI revolutions can be attributed to a higher drying shrinkage because refining increases fibre swelling and hence also drying shrinkage.

The failure strains measured with the subtraction technique are much greater than those measured during a standard tensile test, in which strains were measured which varied from $2.9 \%$ for sheets made from the unrefined pulp to $5.3 \%$ for the sheets made from the stock refined to 6000 PFI revolutions. Similar discrepancies were found by Korteoja et al (Korteoja et al. 1998) who directly measured using image analysis and microscopy, the strain fields in the CD directions of three copy papers during tensile testing. In all cases the local strain around the fracture zone was found to be at least two times larger than the overall average strain across the whole sample, with breaking strains of up to $10 \%$ measured in the fracture zone. Even higher values have been inferred by Yu et al (2002) who investigated the fracture of papers with a range of chemical and mechanical pulp compositions. . From modeling the microscopic damage intensity around the fracture line they estimated strains in the fracture zone ranging from 10 to $20 \%$, much higher than the fracture strain for the sample as a whole (Yu et al. 2002). These results are not necessarily surprising. Paper will fail at its weakest point and the plastic deformation will be at its maximum in the fracture process zone surrounding the crack. However, in a standard tensile test, the size of the fracture process zone is small compared to the overall size of the tensile sample and so the higher strain in the fracture process zone has only marginal influence on the average strain of the whole sample. Span strained under instrument jaws

If the sample is linear elastic, then it is possible to use the stress-strain curves determined by subtraction to estimate the span under the jaws over which slippage is occurring. The method is to use the measured elastic modulus to calculate the strain corresponding to each measured force in the zero-span test. The corresponding displacement is then divided by the strain to arrive at a span. In calculating this span it has been assumed that the full load is applied across this span. If there are any frictional forces, such as was postulated by the Cowan model, then the span estimated by this method will be a lower bound for the distance over which slippage is actually occurring, since the maximum force will not be applied across the width of the slippage zone.

The estimated minimum spans, for testing sheets made from unrefined fibres and fibres refined to 1000 and 3000 PFI revs, are shown in Figure 8. These were calculated by fitting the data from the sheets made from the fibres refined to 3000 PFI revs, to determine an elastic modulus. This was only done from 0 to $120 \mathrm{kNm} / \mathrm{kg}$ where the stress-strain data is approximately linear. The fitted modulus, with a value of $1677 \mathrm{kNm} / \mathrm{kg}$, is shown in Figure 7. As the stress-strain data for the sheets made from the unrefined fibres and from the fibres refined to 1000 and $3000 \mathrm{PFI}$ revs are coincident over this range, the same fitted elastic modulus was applied to all three sets of data. The span during testing sheets made from fibres refined to 6000 PFI revs was not calculated, because this data shows considerable apparent plastic deformation, which make it difficult to assess the minimum span.

When the data in Figure 8 are examined, it can be seen that the papers made from the unrefined fibres and the fibres refined to 1000 PFI revs show approximately the same behaviour. Both show an initial span of $200 \mu \mathrm{m}$ at the start of the test, which stays approximately constant until mid-way through the test, where it then starts to gradually increase. The span under the jaws is not calculated until sample fracture as the non-linear mechanical properties close to fracture make it difficult to analyse. However, for the final 
data points that have been analysed, the calculated span under the jaws has risen to $300 \mu \mathrm{m}$. The sheets made from the fibres refined to 3000 PFI revs show similar behaviour, except that the calculated spans are 50-60 $\mu \mathrm{m}$ higher across the range of the test. A mechanism of stress transfer, which might fit the data in Fig. 8 is one in which there is a limited zone of slippage, possibly with associated frictional forces, with the remainder of the force being transferred at a sticking point behind the jaw. The point at which this surface layer transfers force seems to move somewhat further away from the jaw edge as the test proceeds, possibly by some type of stick-slip mechanism. The constant span under the jaws for the first half of the test is not easy to explain. Certainly, the data is not consistent with the Cowan friction model, which posits that the span under the jaw should be proportional to $F_{L}^{2}$. One possible explanation for the calculated constant span may be if the jaws are slightly misaligned, such that the pressure on the sample increases, moving in from the edge of the jaw. The increase in the calculated span, for the sheets made from pulp refined to 3000 PFI mill revs, might then be a consequence of the sheet density increasing with refining. This could then change the point at which the pressure is large enough to hold the sheet. Certainly more work needs to be done to understand the stress-transfer mechanism under the jaw in zero and short span tests.

\section{CONCLUSION}

A new method has been developed for measuring the stress-strain curve of the paper short span by subtracting an average zero-span displacement-load curve from a short span displacement-load curve. If the sample is well bonded and the free span is small compared to the fibre length, then the free span stress-strain curve is independent of span. The new method was used to measure the effect of refining on paper made from an unbleached, neverdried commercial Scandinavian softwood kraft. The short span stress-strain curve of the paper made from the unrefined pulp was almost entirely linear-elastic. After refining to 6000 PFI revolutions, the short span stress-strain curve showed a reduced modulus, a lower yield stress and greatly increased plastic deformation. One of the stress-strain curves was used to calculate the minimum span being strained under the jaw during a test. The span was found to be approximately constant at the start of a test and then to begin to slowly increase from the mid-point of the test onwards. The range of spans was 200-350 $\mu \mathrm{m}$. These results suggest that the stress transfer to the sample under the jaws may be exhibiting some kind of stick-slip behaviour.

\section{ACKNOWLEDGEMENT}

We acknowledge SCA Graphic Research AB for their support for this research. We also acknowledge Anneli Neumann and Annika Sedin for their handsheet preparation and testing, Sten Larsson for his assistance with the data acquisition, Rickard Boman and Tomas Unander for their assistance with Matlab programming and Rickard Hägglund for useful discussions.

\section{REFERENCES}

Batchelor, W.J. (1999): Determination of Load-Bearing Element Length in Paper Using Zero/Short Span Tensile Testing. In "TAPPI International Paper Physics Conference" TAPPI, pp. 247-258.

Batchelor, W.J. (2002): Determination of Load-Bearing Element Length in Paper Using Zero/Short Span Tensile Testing, To be published in TAPPI Journal 1(9). 
Cowan, W.F. (1975): "Short span tensile analysis". Montreal, Canada, Pulmac Instruments.

Jayaraman, K. and Kortschot, M.T. (1998): Closed-form network models for the tensile strength of paper - a critical discussion, Nordic Pulp Paper Res. J. 13(3), 233-242.

Korteoja, M.J., Niskanen, K.J., Kortschot, M.T. and Kaski, K.K. (1998): Progressive damage in paper, Paperi Ja Puu-Paper \& Timber 80(5), 364-372.

Mohlin, U.B., Dahlbom, J. and Hornatowska, J. (1996): Fiber deformation and sheet strength, TAPPI J. 79(6), 105-111.

Page, D.H. (1969): A Theory for the Tensile Strength of Paper, Tappi 52(4), 674-681.

Perez, M. and Kallmes, O.J. (1965): The Role of Fiber Curl in Paper Properties, Tappi 48(10), 601-606.

Seth, R.S. (1999): Zero-span tensile strength of papermaking fibres. In "85th Annual Meeting, PAPTAC" PAPTAC, Montreal, pp. A161-A173.

Seth, R.S. (2001): Zero-span tensile strength of papermaking fibres, Paperi Ja Puu-Paper \& Timber 83(8), 597604.

Van Den Akker, J.A., Lathrop, A.L., Voelker, M.H. and Dearth, L.R. (1958): Importance of Fiber Strength to Sheet Strength, Tappi 41(8), 416-425.

Yu, Y., Kettunen, H. and Niskanen, K.J. (2002): Connection Between the Damage Profile and Cohesive StressWidening Curve of Paper, Journal of Pulp and Paper Science 28(5), 176-181. 
Figure 1 Forces on paper between jaws of tensile tester

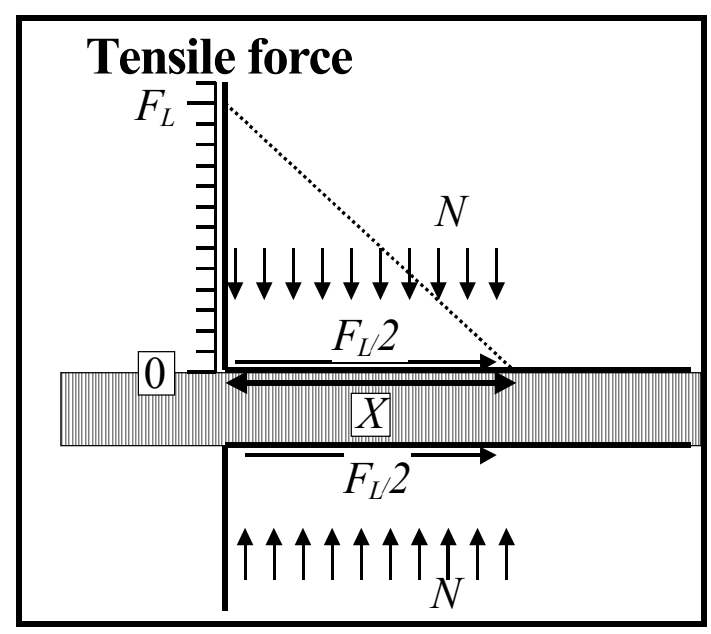


Figure 2 Raw force vs displacement curves for 24 zero-span tests on the sheets made from an unbleached softwood kraft beaten to 6000 PFI revs. Each test exhibits an inflexion point around $12 \mu \mathrm{m}$ due to an initial slack in the specimen or the test equipment

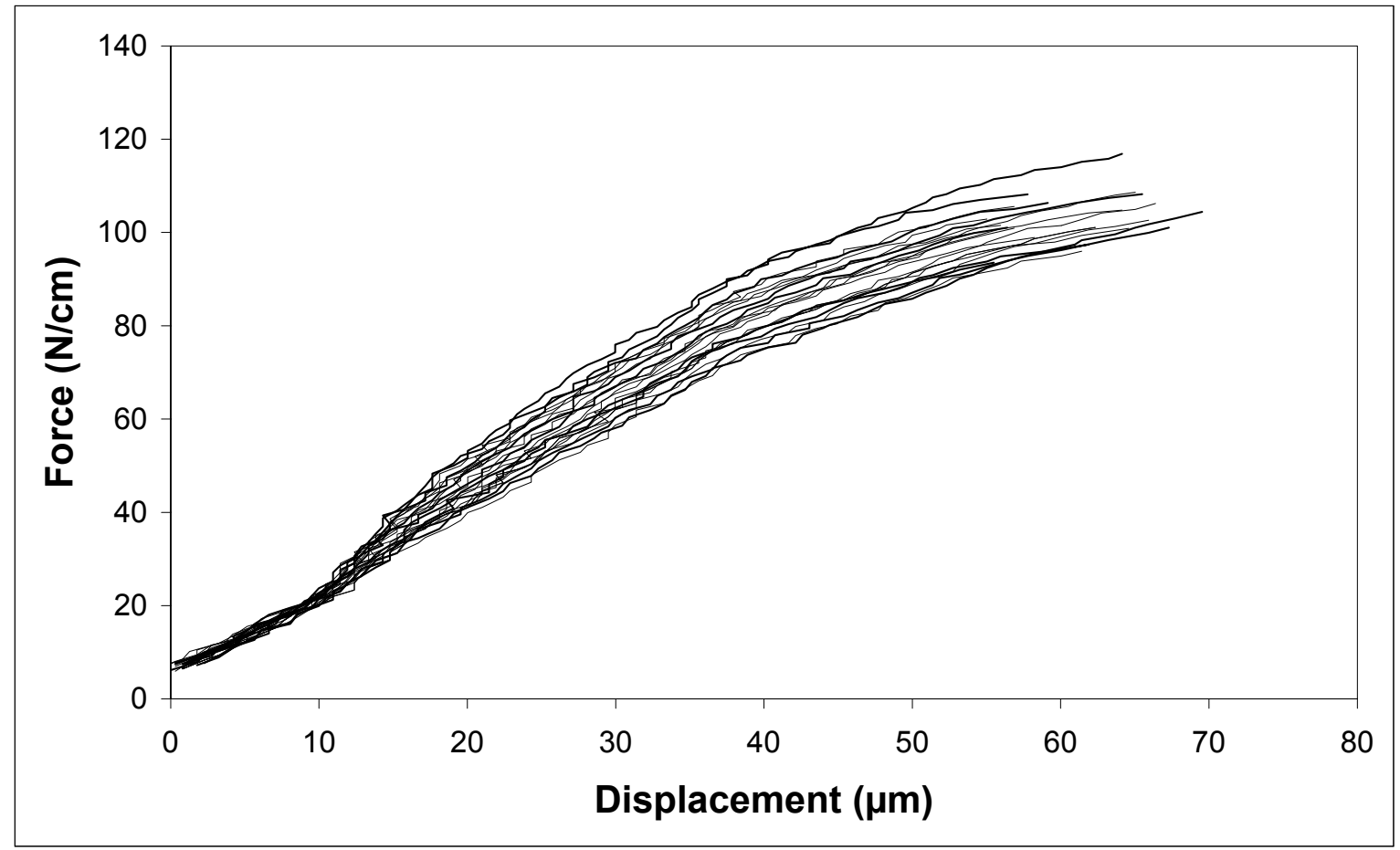


Figure 3 Smoothed and averaged force displacement curves for an unbleached softwood kraft beaten to 6000 PFI mill revs and freely dried.

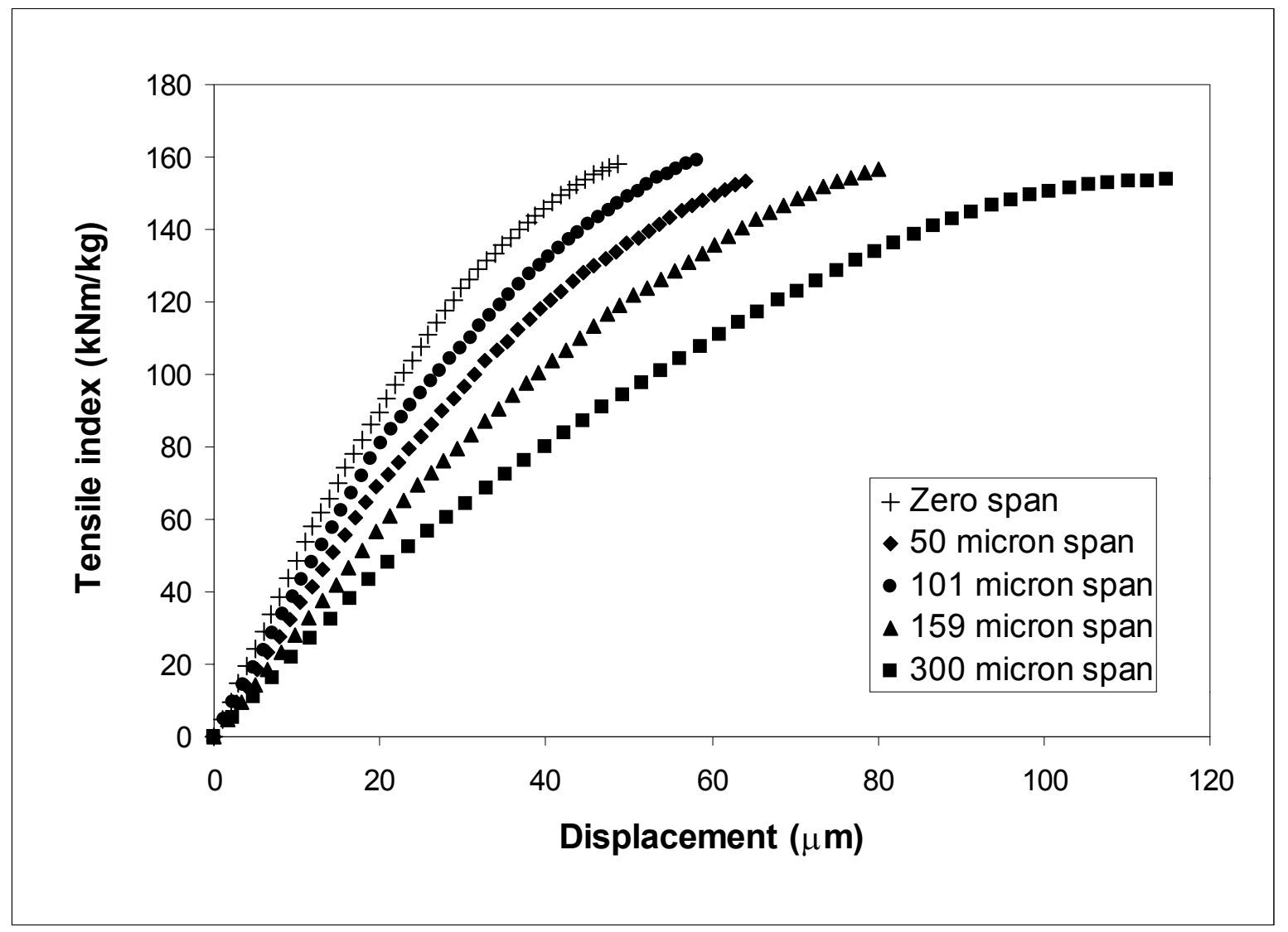


Figure 4 Stress-strain curves measured by subtraction for an unbleached softwood kraft pulp beaten to 6000 PFI revs.

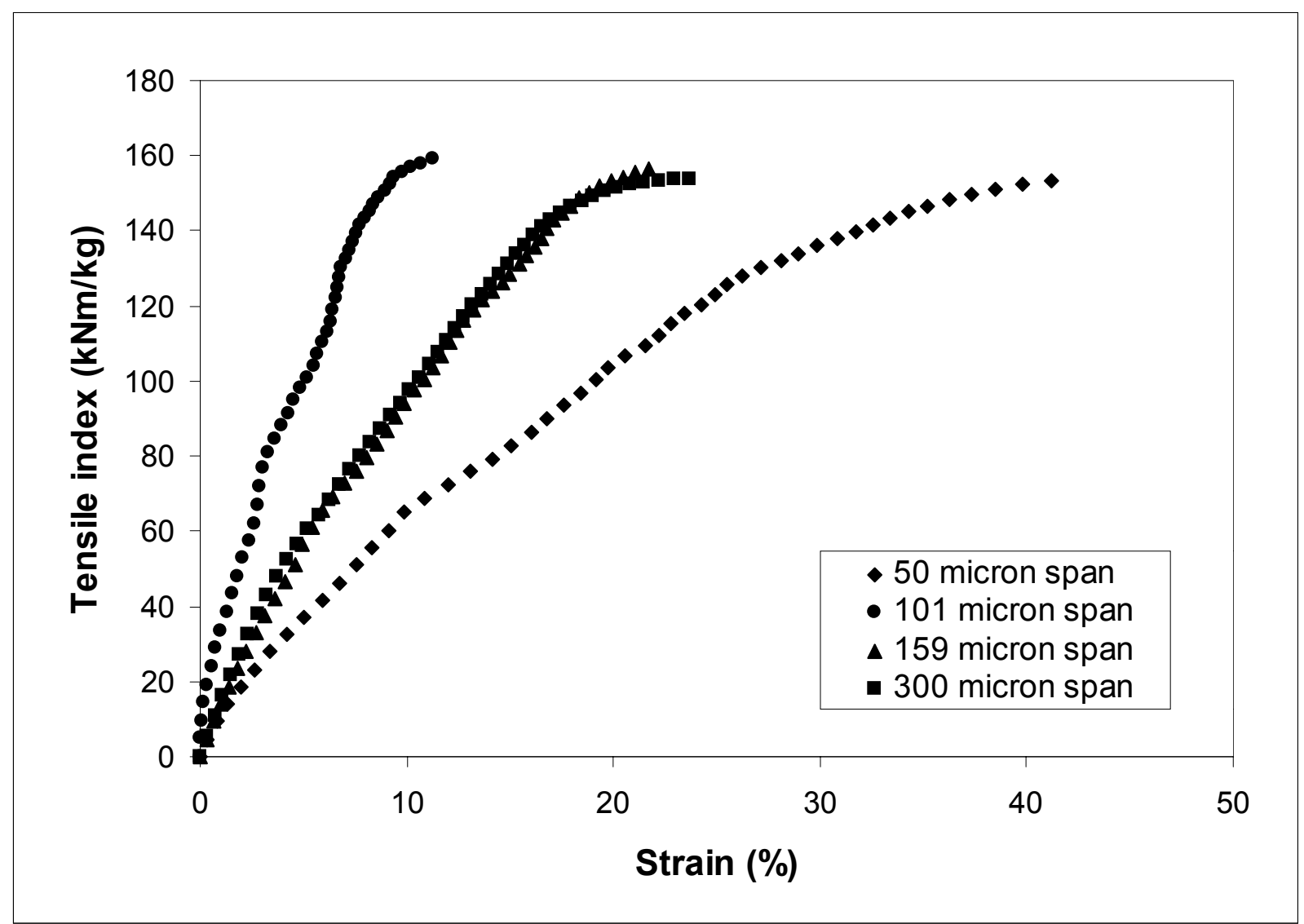


Figure 5 Stress-strain curves measured by subtraction for an unbleached, unbeaten softwood kraft pulp.

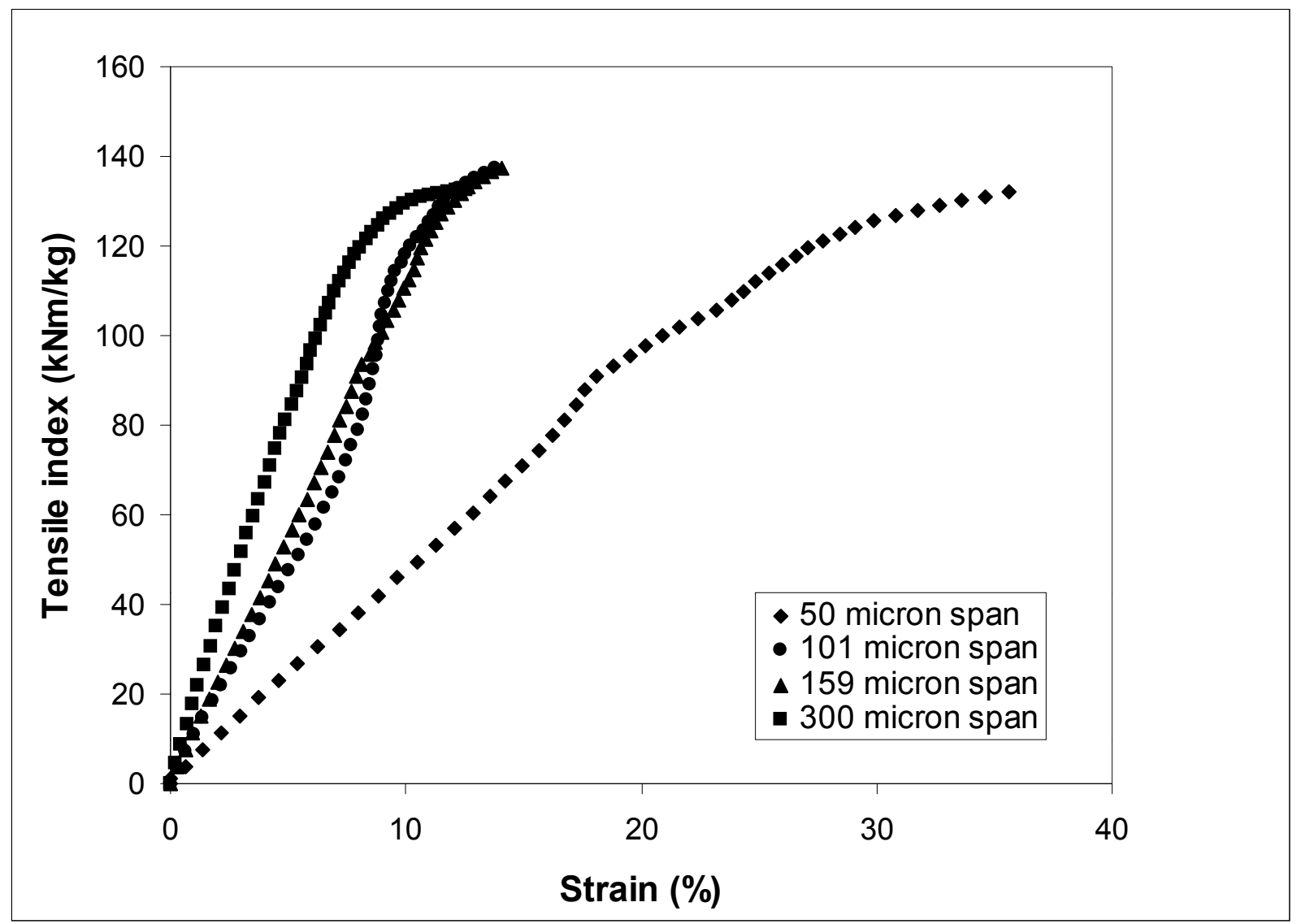


Figure 6 Stress-strain curves measured by subtraction for a unbleached, softwood kraft pulp beaten to 3000 PFI revolutions.

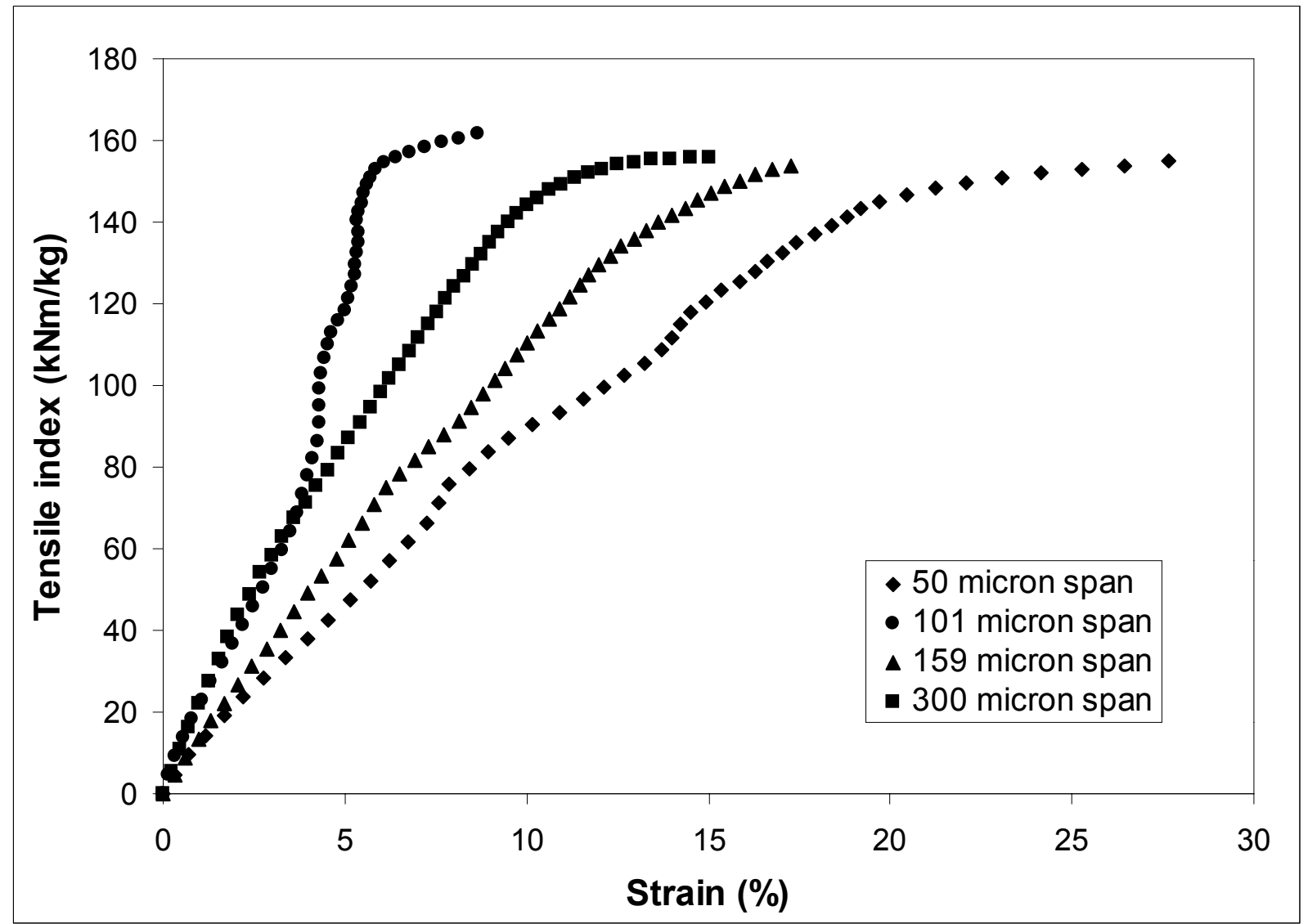


Figure 7 Stress-strain curves as a function of PFI mill revolutions. Curves were measured by subtracting zero-span data from the $300 \mu \mathrm{m}$ span data. The line shows the elastic modulus fitted to the data from the sheets refined to 3000 PFI mill revolutions.

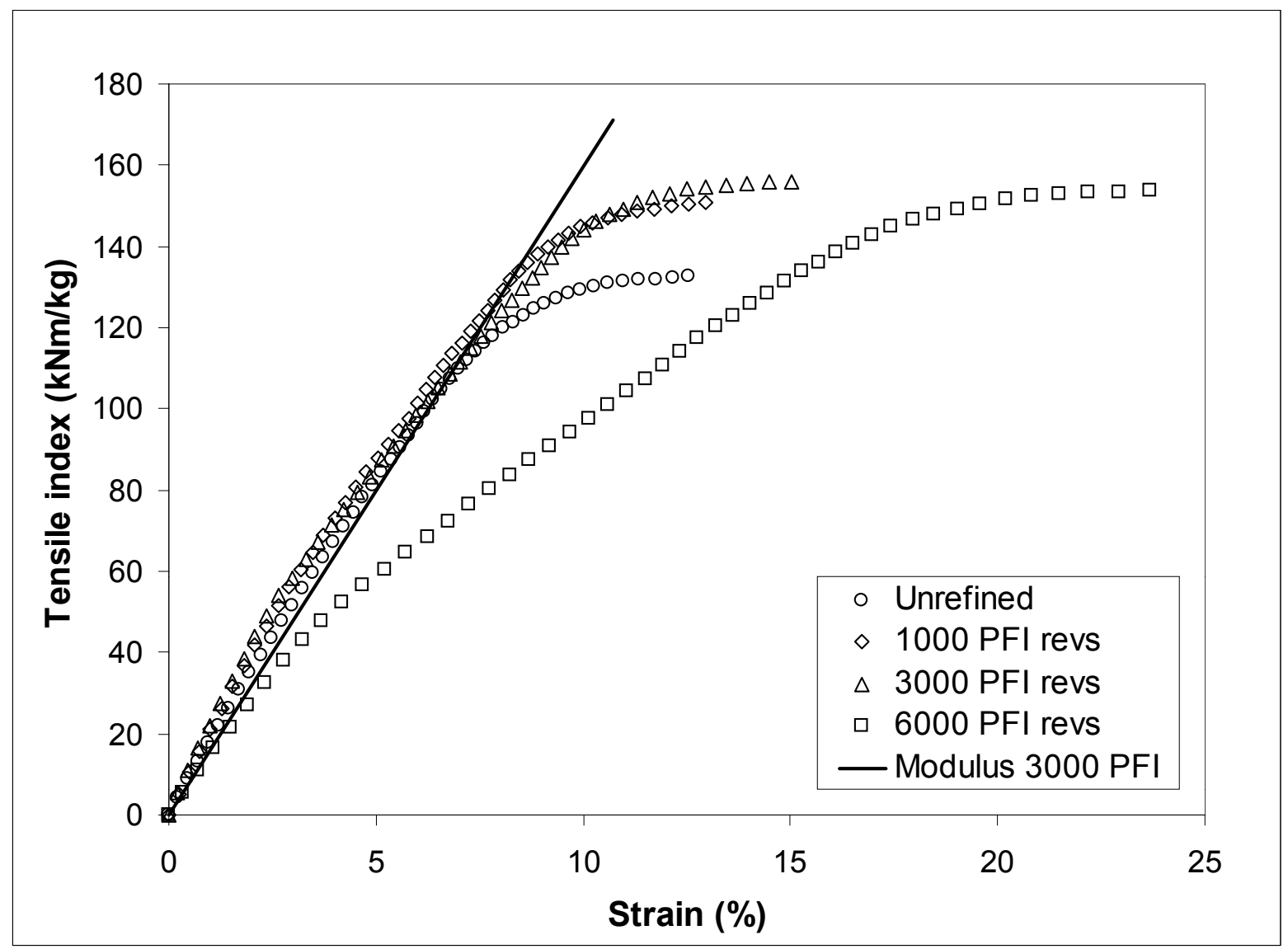


Figure 8 Span under jaw estimated from paper cross-section stress-strain curves and measured forces and displacements during the zero-span test.

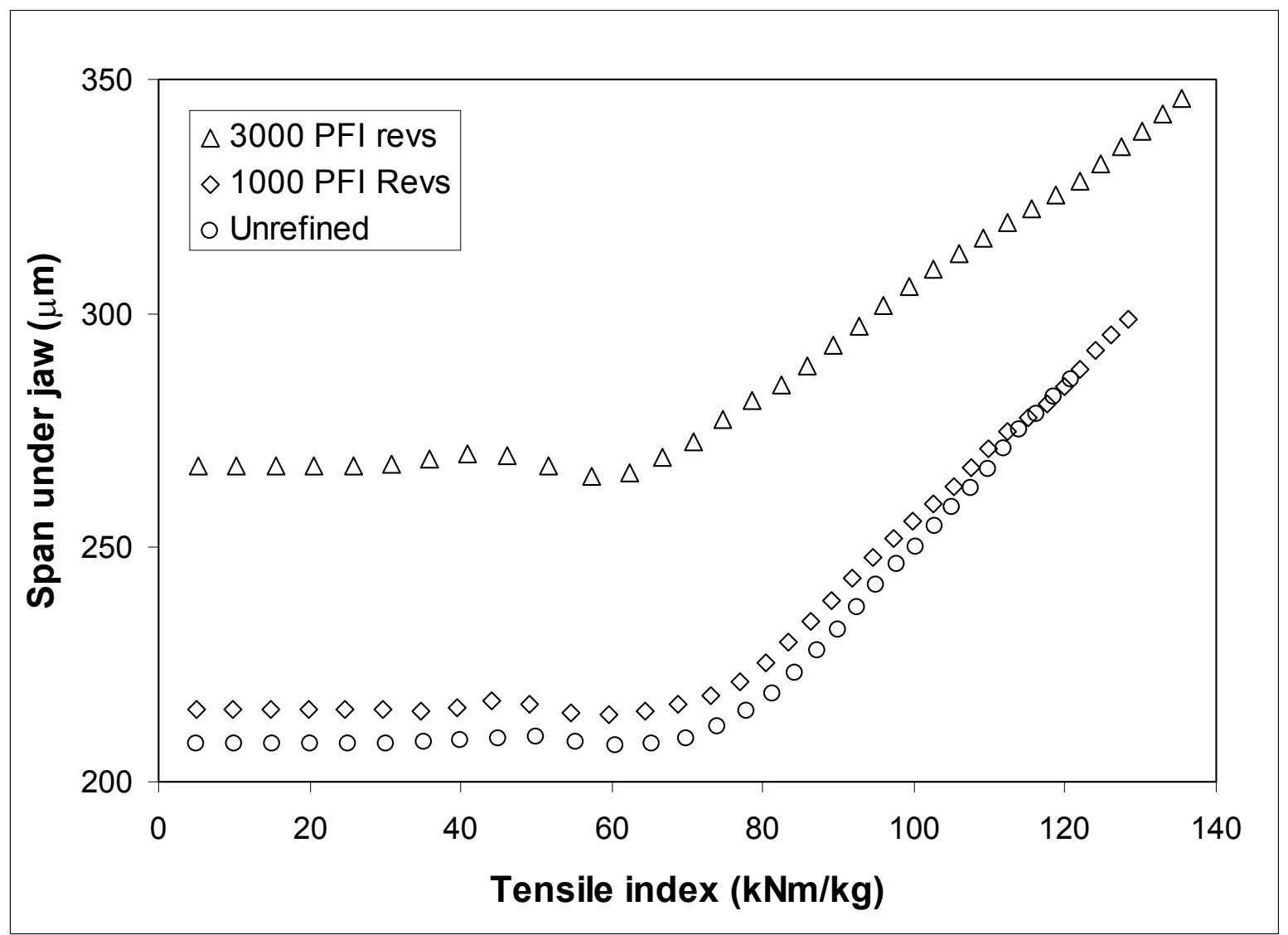


Table 1 Summary of zero/short span and conventional test data

\begin{tabular}{|c|c|c|c|c|c|}
\hline $\begin{array}{c}\text { Beating: } \\
\text { PFI revol- } \\
\text { utions }\end{array}$ & $\begin{array}{c}\text { Span } \\
\mu \mathbf{m}\end{array}$ & $\begin{array}{c}\text { Displacement } \\
\mathbf{a t ~ b r e a k} \\
\mu \mathbf{m}\end{array}$ & $\begin{array}{c}\text { Zero/ Short Span } \\
\text { Tensile Index } \\
\mathbf{k N m} / \mathbf{k g}\end{array}$ & $\begin{array}{c}\text { Tensile Strength } \\
\mathbf{( k N m / k g )}\end{array}$ & $\begin{array}{c}\text { Stretch at } \\
\text { Break (\%)- } \\
\text { Tensile test }\end{array}$ \\
\hline 0 & 0 & $32 \pm 3.0$ & $143.9 \pm 5.8$ & 39.3 & 2.9 \\
\hline 0 & 50 & $44 \pm 2.7$ & $132.0 \pm 8.0$ & 39.3 & 2.9 \\
\hline 0 & 100.7 & $42 \pm 2.8$ & $137.3 \pm 8.6$ & 39.3 & 2.9 \\
\hline 0 & 159.8 & $50 \pm 3.3$ & $137.4 \pm 7.5$ & 39.3 & 2.9 \\
\hline 0 & 300 & $64 \pm 6.6$ & $132.5 \pm 5.6$ & 39.3 & 2.9 \\
\hline 1000 & 0 & $33 \pm 4.0$ & $146.7 \pm 9.1$ & 70.3 & 4.4 \\
\hline 1000 & 50 & $41 \pm 2.8$ & $155.7 \pm 6.4$ & 70.3 & 4.4 \\
\hline 1000 & 100.7 & $47 \pm 4.3$ & $154.6 \pm 9.4$ & 70.3 & 4.4 \\
\hline 1000 & 159.8 & $54 \pm 3.3$ & $147.9 \pm 6.9$ & 70.3 & 4.4 \\
\hline 1000 & 300 & $72 \pm 6.9$ & $150.7 \pm 6.9$ & 70.3 & 4.4 \\
\hline 3000 & 0 & $42 \pm 4.9$ & $159.3 \pm 6.2$ & 87.9 & 4.5 \\
\hline 3000 & 50 & $52 \pm 4.4$ & $154.9 \pm 8.5$ & 87.9 & 4.5 \\
\hline 3000 & 100.7 & $51 \pm 3.5$ & $161.6 \pm 8.9$ & 87.9 & 4.5 \\
\hline 3000 & 159.8 & $65 \pm 5.1$ & $153.9 \pm 6.9$ & 87.9 & 4.5 \\
\hline 3000 & 300 & $84 \pm 6.4$ & $156.0 \pm 7.0$ & 87.9 & 4.5 \\
\hline 6000 & 0 & $49 \pm 4.4$ & $158.1 \pm 7.7$ & 97.8 & 5.3 \\
\hline 6000 & 50 & $64 \pm 4.2$ & $153.3 \pm 8.8$ & 97.8 & 5.3 \\
\hline 6000 & 100.7 & $58 \pm 3.8$ & $159.0 \pm 6.4$ & 97.8 & 5.3 \\
\hline 6000 & 159.8 & $80 \pm 4.1$ & $156.6 \pm 9.6$ & 97.8 & 5.3 \\
\hline 6000 & 300 & $115 \pm 10.4$ & $153.9 \pm 6.2$ & 97.8 & 5.3 \\
\hline
\end{tabular}

